\title{
Assessing the Benefits of NASA Category 3, Low Cost Class C/D Missions
}

\author{
Robert Bitten \\ The Aerospace Corporation \\ 2310 E. El Segundo Blvd. \\ El Segundo, California 90009 \\ (310) 336-1917 \\ robert.e.bitten@aero.org
}

\author{
Steve Shinn \\ Goddard Space Flight Center \\ 8800 Greenbelt Rd., Code 400 \\ Greenbelt, Maryland 20771 \\ (301) 286-5894 \\ stephen.a.shinn@nasa.gov
}

\author{
Eric Mahr \\ The Aerospace Corporation \\ 400 E Street NW \\ Washington, DC 20024 \\ (202) 358-5118 \\ eric.m.mahr@aero.org
}

\begin{abstract}
Category 3, Class C/D missions have the benefit of delivering worthwhile science at minimal cost which is increasingly important in NASA's constrained budget environment. Although higher cost Category 1 and 2 missions are necessary to achieve NASA's science objectives, Category 3 missions are shown to be an effective way to provide significant science return at a low cost. Category 3 missions, however, are often reviewed the same as the more risk averse Category 1 and 2 missions. Acknowledging that reviews are not the only aspect of a total engineering effort, reviews are still a significant concern for NASA programs. This can unnecessarily increase the cost and schedule of Category 3 missions. This paper quantifies the benefit and performance of Category 3 missions by looking at the cost vs. capability relative to Category 1 and 2 missions. Lessons learned from successful organizations that develop low cost Category 3, Class C/D missions are also investigated to help provide the basis for suggestions to streamline the review of NASA Category 3 missions.
\end{abstract}

\section{TABLE OF CONTENTS}

1. INTRODUCTION 1

2. BENEFITS OF CATEGORY 3 MISSIONS ...........................2

3. FAILURE RESULTS SUMMARY.

4. NASA REVIEW PROCESS \& RECOMMENDATIONS FOR STREAMLINING.

5. SUMMARY. 6

ACKNOWLEDGEMENTS 10

REFERENCES 11

BIOGRAPHY. 12

\section{INTRODUCTION}

Category 3 missions are the lowest cost and highest risk missions within NASA's science portfolio. Category 3 missions do, however, provide significant benefit to NASA by providing important science contributions at a low cost. [1][2][3] The success of Category 3 missions becomes even more important at this time when NASA budgets are becoming more and more restrictive. The continued success of Category 3 missions will be important to NASA's future.

NASA has developed a set of guiding documents to provide different requirements and governing principles for missions of differing levels of criticality. NASA Procedural Requirement (NPR) 7120.5E defines different categories of missions based on their priority to NASA's strategic goals

978-1-4673-1813-6/13/\$31.00 @2013 IEEE and their relative total mission life cycle cost (LCC), as shown in Table 1. [4] Similarly, NPR 8705.4 defines different classes of missions based upon a variety of factors, as shown in Table 2. [5] The definitions of mission categories and classes allow a distinction between missions in terms of guidelines for development. Although there are clear guidelines for elements such as parts selection and testing requirements, the review requirements for these missions are much more ambiguous. This ambiguity often leads review teams to default to the common practices and extensive requirements of larger missions. As a result, the lower priority, lower cost Category 3, Class $C / D$ missions are being reviewed similar to high priority, higher cost Category $1 / 2$, Class $\mathrm{A} / \mathrm{B}$ missions. A common statement in NASA is "Every mission is Class A by the time it launches". The primary benefit of Category 3 missions is their ability to collect science data at a relatively low cost. By treating Category 3 missions the same as Category 1 or 2 missions, the effort reduces this benefit and provides a substantial burden on the Category 3 mission project team and reduces the benefit to NASA and its stakeholders.

Table 1. Category 1, 2, 3 Definitions from NPR 7120.5E

\begin{tabular}{|c|c|c|c|}
\hline $\begin{array}{l}\text { PRIOR } \overline{T V} \\
\text { [.I.VHE: }\end{array}$ & $\begin{array}{l}18 \mathrm{~B} 8 \\
5860 \mathrm{M}\end{array}$ & $\begin{array}{l}4250 \mathrm{M} \\
1.6 \mathrm{E}-5 \mathrm{~s}\end{array}$ & $\begin{array}{l}\text { EEES } \\
\text { sulis }\end{array}$ \\
\hline IMin & CAT 2 & CAT 2 & CAT 1 \\
\hline M(G) & CAT 3 & CAT 2 & CAT 1 \\
\hline Ixank & CAT 3 & CAT 2 & CAT 1 \\
\hline
\end{tabular}

Table 2. Class A, B, C, D Definitions from NPR 8705.4

\begin{tabular}{|c|c|c|c|c|}
\hline Charncteristif & Ehasy & Gass B & Cilass $\mathrm{C}$ & chuss D \\
\hline $\begin{array}{l}\text { Stratcale } \\
\text { triturty }\end{array}$ & High & High & Medium & L.ow \\
\hline $\begin{array}{c}\text { Mecepinble } \\
\text { Bish }\end{array}$ & $\begin{array}{l}\text { Very } \\
\text { Low }\end{array}$ & Low & Medium & High \\
\hline $\begin{array}{l}\text { Ninghil } \\
\text { Signifturice }\end{array}$ & $\begin{array}{l}\text { Very } \\
\text { High }\end{array}$ & High & Medium & $\begin{array}{l}\text { Low to } \\
\text { Medium }\end{array}$ \\
\hline $\begin{array}{l}\text { Btisston } \\
\text { Eomotosity }\end{array}$ & $\begin{array}{l}\text { Very } \\
\text { High }\end{array}$ & $\begin{array}{l}\text { High to } \\
\text { Medium }\end{array}$ & $\begin{array}{l}\text { Medium } \\
\text { to Low }\end{array}$ & $\begin{array}{l}\text { Medium } \\
\text { to Low }\end{array}$ \\
\hline $\begin{array}{l}\text { Nitesion } \\
\text { Cosi }\end{array}$ & High & $\begin{array}{l}\text { High to } \\
\text { Medium }\end{array}$ & $\begin{array}{l}\text { Medium } \\
\text { to Low }\end{array}$ & Low \\
\hline
\end{tabular}

This paper addresses the benefits of the lower cost Category 3 , Class C/D missions, looks at relative failure rates of 
similar organizations that manage and build Category 3 missions, and provides recommendations for potentially streamlining the review process for Category 3 missions to maintain or improve quality while retaining the benefits of these low cost missions.

\section{BeNEFITS OF CATEgoRy 3 Missions}

\section{Overview}

NASA employs a mix of missions within its portfolio to accomplish desired science objectives. Large Category 1 high priority flagship missions, such as the Hubble Space Telescope, are required to answer unique science questions that only a large scale telescope can address. Other missions, like the Cassini and Galileo Orbiters, are the most cost effective way to operate a large number of scientific instruments orbiting a distant planet. Medium sized Category 2 missions are also important as they can provide focused platforms for science that requires either multiple instruments for simultaneous observations or single medium to large sized instruments that have a unique scientific objective. Less costly Category 3 missions are also necessary, however, to conduct initial observations or fill gaps in knowledge for certain science disciplines. They can also be significantly more focused in their science objectives, yielding a small, but significant scientific result.

\section{Data Collection and Mission Categorization}

To assess the benefit of each category of missions, data were collected for NASA missions launched within the last 15 years. The intent was to do a comparison between Category 1,2 and 3 missions to characterize their mission cost, failure rate and overall benefit. The result is a set of 62 NASA missions listed individually in the Appendix. The missions included in the study represent a wide range of category and class of missions. Figure 1 shows the distribution of the Life Cycle Cost (LCC) in FY12\$ as compared to the category and class of the mission. All real year mission cost data were inflated to fiscal year 2012 dollars (FY12\$) so as to represent, as best as possible, the real year dollar guidance for LCC categories as stated in NPR $7120.5 \mathrm{E}$ and shown in Table 1 . As can be seen, Category 1 missions consist of Class A and B missions, where Category 2 missions consist of a balanced mix of Class $\mathrm{B}$ and $\mathrm{C}$ missions while Category 3 consists of Class $\mathrm{C}$ and $\mathrm{D}$ missions. Category 3 missions include the lower cost half of the Class $\mathrm{C}$ missions launched within the last 15 years.

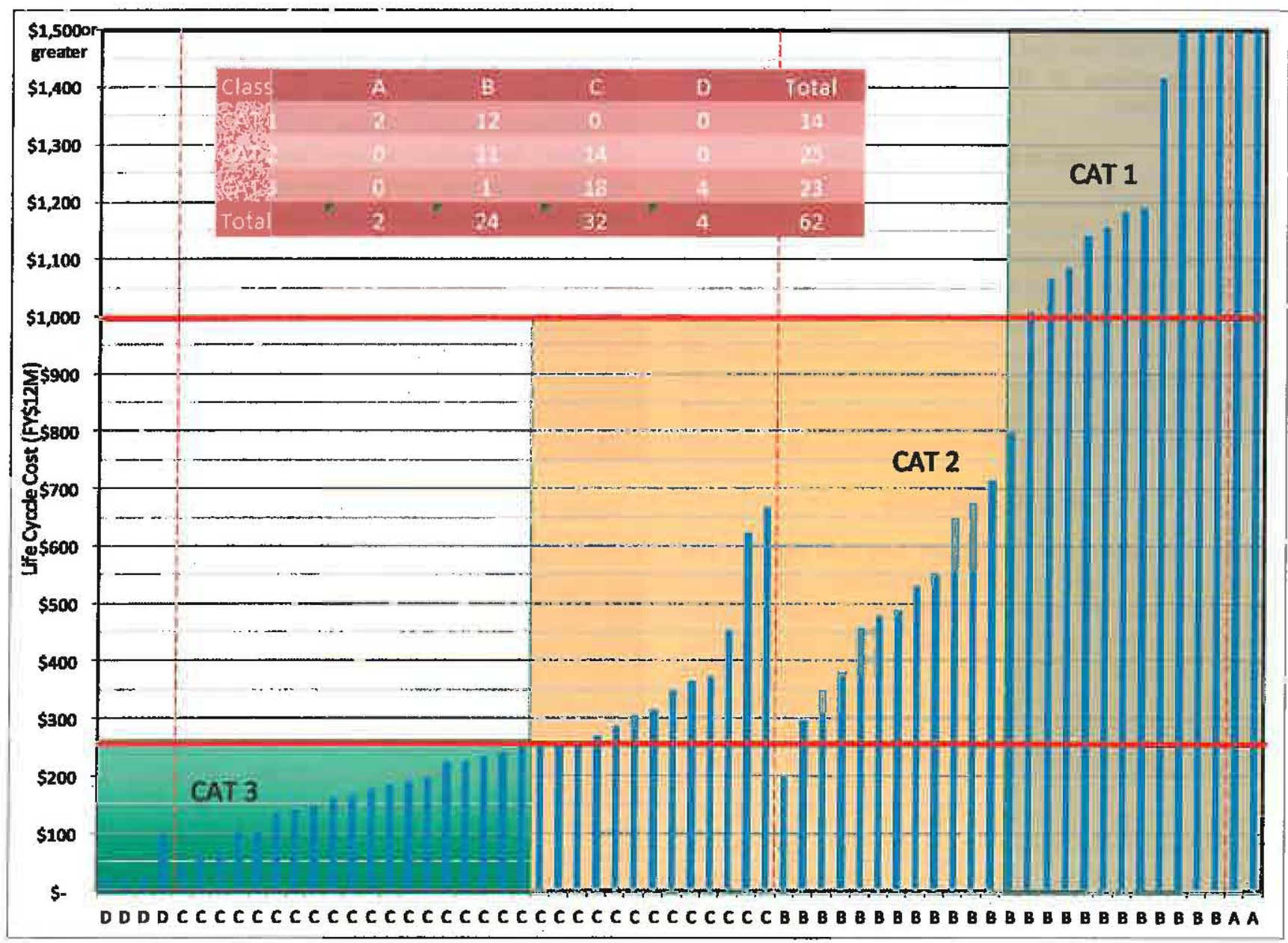

Figure 1 - Life Cycle Cost Characterization of Mission Category \& Class for the Study Data Set 
One of the objectives of the paper was to identify the relative science cost/benefit of each mission class. As such, only NASA Science Missions that meet certain criteria were considered. Missions that have recently been launched but have yet to begin their science missions, such as the Radiation Belt Storm Probes (RBSP) and Nuclear Spectroscopic Telescope Array (NuSTAR) missions, were not considered because their success has yet to be determined at the time of writing. Missions that relied heavily on international contributions, such as the Tropical Rainfall Measuring Mission (TRMM) and CALIPSO missions, were also eliminated from consideration due to the difficulty of assessing foreign costs. Additionally, only full science missions were considered. Therefore "Instrumentonly" science experiments, where the instrument was provided to another organization, were not considered. Technology demonstrators, such as NanoSail-D and Demonstration of Autonomous Rendezvous Technology (DART), were also excluded given that their primary focus is technology demonstration, not science. In addition, operational missions like the Geostationary Operational Environmental Satellite (GOES) series, were excluded in order to focus on more typical, one of a kind NASA science missions.

\section{Cost Categorization}

The average cost for each mission category is shown in Figure 2. Given that one of the primary criteria for categorization of missions per NPR $7120.5 \mathrm{E}$ is cost, the result is as expected with Category 1 missions being substantially higher than Category 2 missions which are more costly than Category 3 missions.

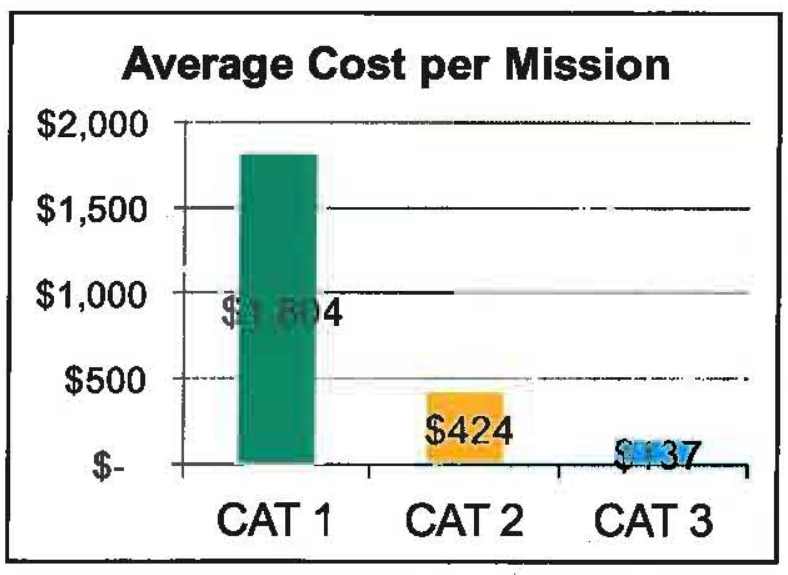

Figure 2 - Average Cost (FY12\$M) per Category

\section{Failure Rate Categorization}

The average failure rate for each mission category was also calculated, as shown in Figure 3 with Category 3 missions, being mostly comprised of Class $\mathrm{C}$ and $\mathrm{D}$ missions, experiencing a much higher failure rate than Category 1 or 2 missions. A mission failure is defined as a launch vehicle or spacecraft failure. An interesting note, which is discussed more in Section 3, is that Category 3 missions also have a much higher non-confirmation rate.

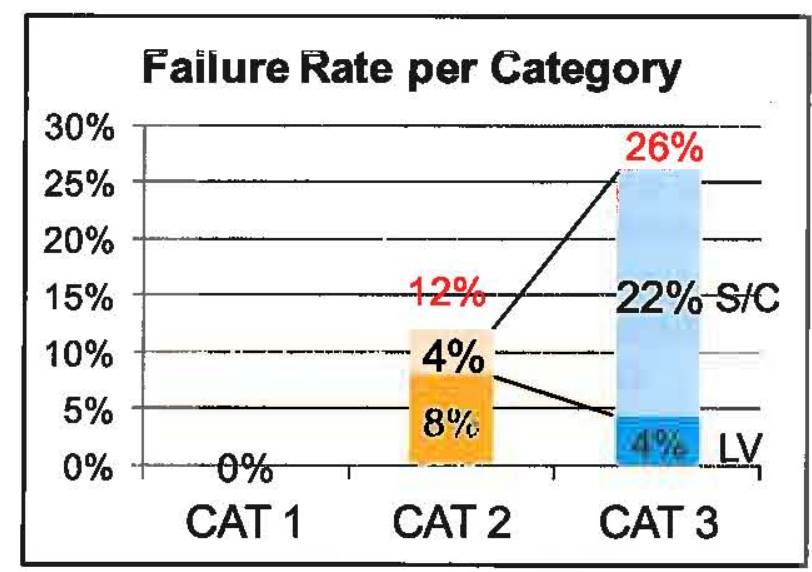

Figure 3 - Average Failure Rate per Category

\section{"Science Return" Categorization}

Assessing the benefit of a science mission is typically very subjective as the "value" to one scientist for any given data returned will not be the same as to another scientist in another discipline. There is no perfect way to judge scientific instrument value. To assess the benefit for the purposes of the study, two "science return" metrics were investigated to provide an objective measure of benefit. The first "science return" metric calculates the number of instruments operating over their lifetime and was originally proposed as an objective quantification of overall science value. [6] In addition, a second metric was defined to look at the total data returned from all science instruments over the lifetime of the mission. Other metrics were considered but found to have certain issues that were hard to overcome. For example, the number of papers published by mission scientists was considered but was believed to disproportionately favor large, prolific teams that publish multiple papers versus a small team that publish a few, very significant papers. Similarly, a metric based on the number of "significant" findings that resulted from the mission would be challenging to use given that the term "significant" is very subjective and difficult to quantify. It has also been suggested that the science value of an instrument is proportional to its mass. This metric suggests, however, that planetary missions are inherently less valuable than Earth orbiting missions because planetary missions typically have much less payload mass due to the difficulty of getting its payload to its final destination. Combined, the two proposed metrics should provide a reasonable assessment of the benefit of the different classes.

The first proposed metric uses the number of instruments on-board the satellite multiplied by the length of time the instruments take data at their final destination and is measured in terms of "instrument-months." [6] Multiplying by the duration that the instrument operates provides a surrogate for the quantity and depth of 
information gathered by the instrument. The proposed metric also accounts for full and partial mission failures because the failed mission's instrument duration of operation, and corresponding science return, would be zero.

When this metric is applied to the dataset used for this study, the results are as expected, with Category 1 missions returning more science than Category 2 missions which return more value than Category 3 missions, the results of which are shown in Figure 4.

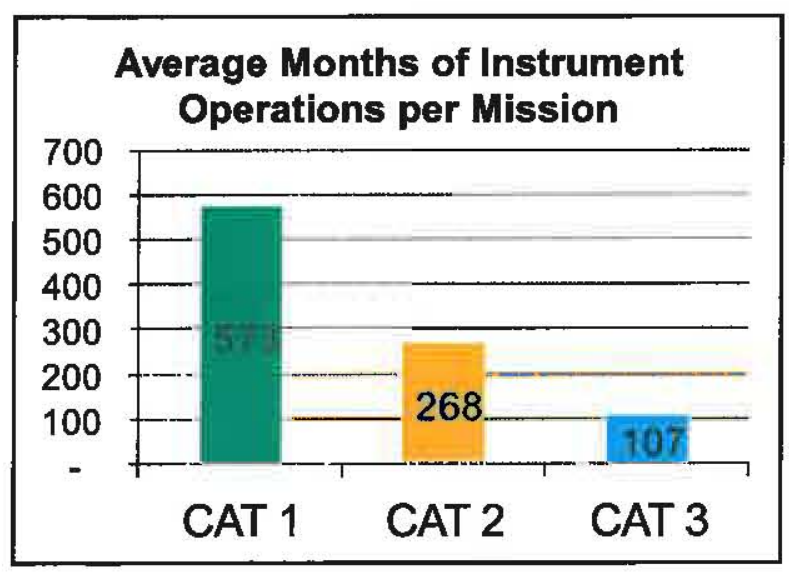

Figure 4 - Average Instrument Month per Mission

The second metric closely parallels the instrument-months metric but utilizes the instrument data rate and operating durations to calculate the data returned from the mission data set. When this metric is applied, the results are also as expected, with Category 1 missions returning more data than Category 2 missions which return significantly more data than Category 3 missions, the results of which are shown in Figure 5.

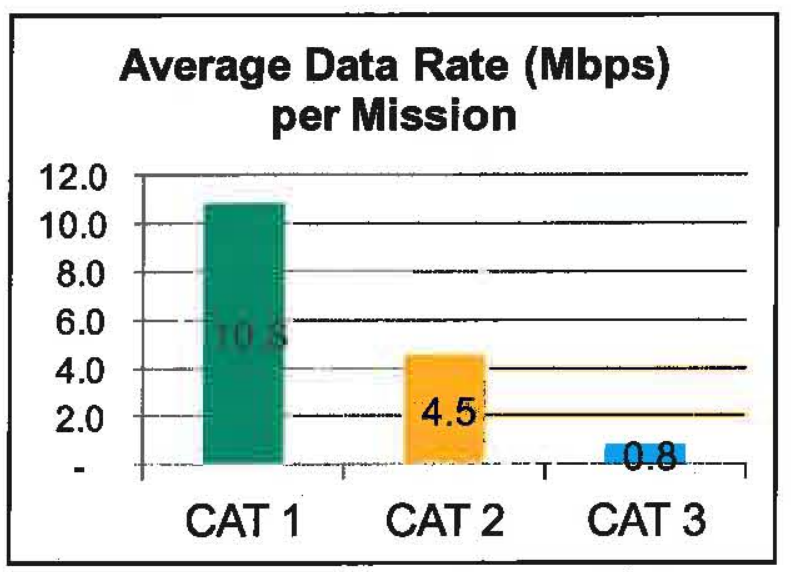

Figure 5 - Average Data Rate per Mission

\section{"Science Return" Metrics Caveats \& Limitations}

These metrics provide a different perspective of value for each mission class but each has its limitations. A primary assumption of the first metric is that all instruments provide equal science value. The basic rationale for this assumption is that each instrument is placed on-board a satellite to achieve a specific scientific objective and that all scientific objectives are considered of equal value. The obvious limitation of such a metric is that all instruments are not created equal. The metric itself treats each instrument the same even though a very sophisticated imaging radar instrument is much more complex to develop than a simple magnetometer. It could be argued, however, that the value to the scientist utilizing the magnetometer data is the same as the scientist utilizing the data from a radar image as each is answering a relevant science question with the data obtained.

The primary assumption of the second metric is that every data bit generated by a science mission is of the same value as any other bit from another mission. This a limiting assumption, however, as an instrument that collects significant amounts of data, such as a Synthetic Aperture Radar (SAR), would be deemed inherently more valuable than instruments that collect less data.

The value of each instrument that NASA launches cannot be understated. NASA employs a severely competitive science selection process using a peer review board of scientists to select the most valuable science from all proposals submitted. Table 3 identifies that, over the last 15 years of Small Explorer (SMEX) and the no longer existing University Explorer (UNEX) proposals submitted, only the top $6 \%$ were selected for implementation. Given the number of proposals submitted and the thoroughness of the evaluation process, it is believed that the science of these missions is of the best that can fit within the cost constraints. Additionally, given that mass, power and volume resources on a spacecraft are always tight and extremely valuable; each instrument has to "buy" its way onto the spacecraft such that the selection of each is warranted. For those missions that can be implemented within Category 3 funding constraints, the competitive process achieves high value science with the selected missions and instruments.

Table 3. NASA SMEX/UNEX Program Selections

\begin{tabular}{|c|ccc|}
\hline Year & $\begin{array}{c}\text { Full } \\
\text { Proposals } \\
\text { Sinbmiticed }\end{array}$ & $\begin{array}{c}\text { Number of } \\
\text { Missions } \\
\text { Sclected }\end{array}$ & Selectod Missions \\
\hline 1997 & 40 & 2 & RHESSI, GALEX \\
1998 & 29 & 2 & CHIPS, IMEX \\
1999 & 33 & 2 & AIM, SPIDR \\
2003 & 29 & 2 & IBEX, AIM \\
2007 & 32 & 2 & IRIS, GEMS \\
\hline Total & 163 & 10 & $6 \%$ Selected \\
\hline
\end{tabular}

\section{"Science Value" Cost Effectiveness Metrics}

The results shown in Figures 2 through 5 are as expected; although Category 1 and 2 missions are more expensive than Category 3 missions, they also fail less often and provide more science return per mission. This is a straightforward outcome given that Category 3 missions are made up of less reliable Class $C$ and Class D missions while the reduced scope required to meet funding guidelines limit 
the number of instruments and years of operations thereby reducing the science return, as indicated by the instrumentmonths and data returned metric.

Given that Category 3 missions are shown to provide such little science return and fail more often, why should Category 3 missions be attempted at all? The answer lies as being cost effective building blocks for future discoveries. Given that science return has been defined with instrumentmonths or as data returned, it is a simple task to divide the two proposed metrics by the total mission LCC to determine the cost-effectiveness of each mission. When viewed this way, these cost-effectiveness metrics measure the mission's "bang for the buck" or the amount of science gathered per dollar. This cost-effectiveness approach can be extended to each mission class by summing the total instrument-moths or data returned for missions in a given class and dividing by the total LCC of the missions in that class. Computing these values, the data presented indicates that Category 3 missions are either the most cost effective category, as shown in Figure 6 relative to instrument months per dollar, or essentially equally as cost-effective, as shown in Figure 7 based on the data returned per dollar.

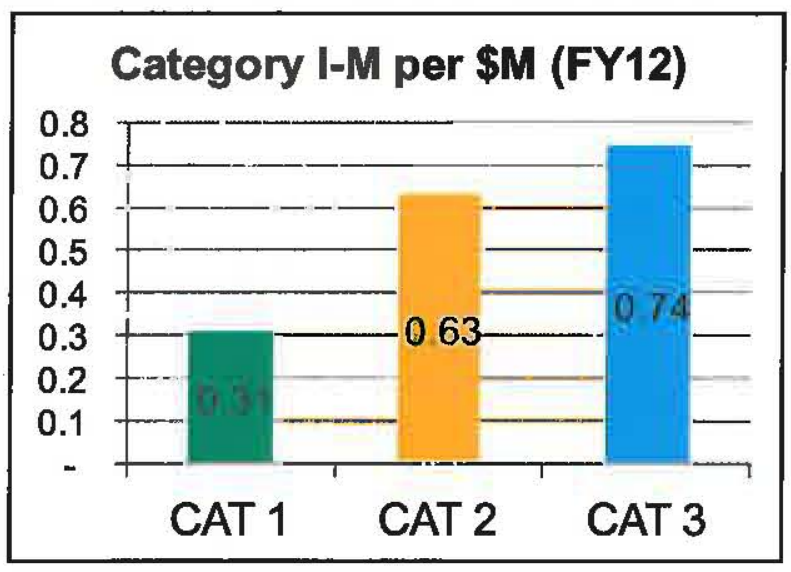

Figure 6 - I-M Science Mission Cost Effectiveness

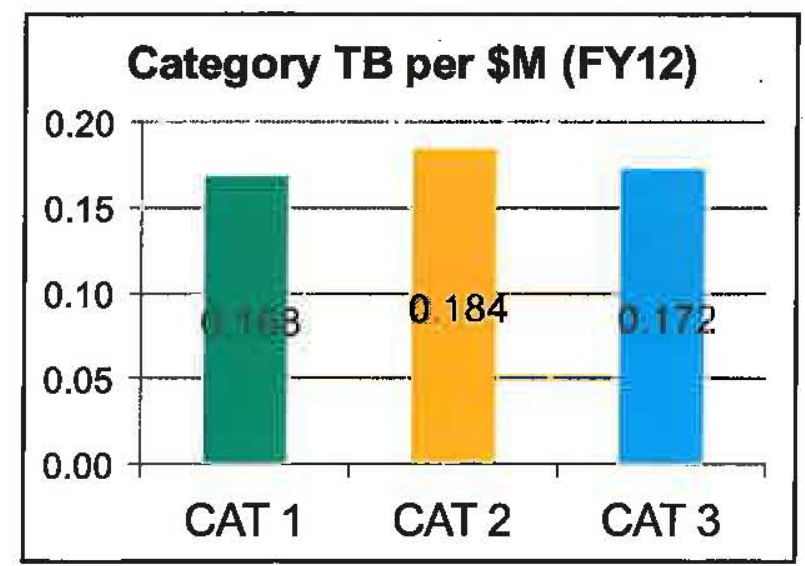

Figure 7 - TB Science Mission Cost Effectiveness

As stated previously, there are certain scientific objectives that cannot be accomplished given the funding and subsequent size and complexity constraints of a Category 3 mission. For those scientific objectives that can be implemented within the constraints of a Category 3 mission, however, the two metrics developed indicate that the mission implementations seem to provide a fairly cost effective acquisition strategy. The data indicates that Category 3 missions can serve as a cost-effective, critical building block for a balanced portfolio of missions.

\section{FAILURE RESUlts Summary}

As shown previously in Section 2.4, satellite failures have occurred more often in Category 3 missions than in Category 1 and 2 missions. As shown in Table 4, the majority of these failures have been in the satellite flight system. In addition, Category 3 missions have a greater probability of being non-confirmed. Of the eight missions that were cancelled or not confirmed from 1997 to 2011, five of those missions were Category 3 missions. Given the 23 Category 3 missions launched, the five missions represent an $18 \%$ cancellation rate (i.e., 5 out of 28 total missions) which is significant. Combined with the failure statistics, this represents a probability of cancellation or failure for missions that are selected on the order of $39 \%$. A further breakdown of the missions that have failed and the possible cause of failure or cancellation/non-confirmation are provided in Table 5. More detail on each mission is contained in the Appendix.

Table 4. Causes of Mission Failure

\begin{tabular}{|c|c|c|c|c|c|}
\hline Giteguny & $\begin{array}{l}\text { IN } \\
\text { Eailinte= }\end{array}$ & $\begin{array}{l}800 \\
\text { Falintse. }\end{array}$ & $\begin{array}{l}\text { Nisgions: } \\
\text { cminet }\end{array}$ & $\begin{array}{l}\text { Totril } \\
\text { Misilans }\end{array}$ & $\begin{array}{c}\text { Total } \\
\%\end{array}$ \\
\hline ENT & 0 & 0 & 1 & 15 & $6.7 \%$ \\
\hline CNT2 & 2 & 1 & 2 & 27 & $18.5 \%$ \\
\hline $\operatorname{cect} 3$ & 1 & 5 & 5 & 28 & $39.3 \%$ \\
\hline
\end{tabular}

Table 5. Causes of Mission Failure

\begin{tabular}{|c|c|c|c|c|}
\hline Mrission & circsosy & $\begin{array}{l}\text { Fature } \\
\text { Rruson: }\end{array}$ & Year & Relerentes \\
\hline Lewis & CAT 3 & Spacecraft & 1997 & 7,8 \\
\hline WIRE & CAT 3 & Spacecraft & 1999 & 9 \\
\hline TERRIERS & CAT 3 & Spacecraft & 1999 & 10 \\
\hline MCO & CAT 3 & Spacecraft & 1999 & 11 \\
\hline MPL & CAT 2 & Spacecraft & 1999 & 12 \\
\hline CONTOUR & CAT 3 & Spacecraft & 2002 & 13 \\
\hline Clark & CAT 3 & Cancelled & 1998 & 14 \\
\hline ST-4 & CAT 2 & Cancelled & 1999 & 15,16 \\
\hline $\mathrm{VCL}$ & CAT 3 & Cancelled & 2001 & 17 \\
\hline IMEX & CAT 3 & Cancelled & 2001 & 18 \\
\hline FAME & CAT 2 & Cancelled & 2002 & 19 \\
\hline SPIDR & CAT 3 & Cancelled & 2002 & 20 \\
\hline CATSAT & CAT 3 & Cancelled & 2002 & 21 \\
\hline SIM & CAT 1 & Cancelled & 2008 & 22 \\
\hline QuikTOMS & CAT 3 & LV & 2001 & 23 \\
\hline
\end{tabular}




\begin{tabular}{|lllll|}
\hline OCO & CAT 3 & LV & 2009 & 24 \\
\hline Glory & CAT 2 & LV & 2011 & 25 \\
\hline
\end{tabular}

\section{NASA REVIEW PROCESS \& RECOMMENDATIONS FOR STREAMIINING}

\section{Overview}

NPR 8705.4 provides guidance for the distinction of Class A, B, C and D missions for a variety of different elements. As a previously described, Category 3 missions are usually covered by Class C \& D guidance. Although NPR 8705.5 provides good guidance on many aspects of mission development, such as testing, parts/materials, safety, reliability, risk management, etc., relative to Class A, B, C and $\mathrm{D}$ missions, there is very little guidance relative to the implementation of reviews. As shown in Figure 8, guidance for reviews is provided in one line. Although this line provides general guidance for overall reviews, it does not address specific requirements for reviews at a lower level.

NPR $7120.5 \mathrm{E}$ also provides some guidance relative to Category 1, 2 and 3 mission, as shown in Figure 9 and states that NASA Centers have the sole Technical Authority for Category 3 missions. Given that is the case, Center
Directors can work with the Mission Directorate Associate Administrator (MDAA) to identify an acceptable review approach. Although Category 3 reviews are governed by NASA Centers, current policy does not provide good, universal guidance on the streamlining of reviews for Category 3 missions. [26]

An example of the growing review requirements for Category 3 missions can be seen by the experience of the Small Explorer (SMEX) Aeronomy of Ice in the Mesosphere (AIM) mission. Initially the AIM team, as part of their proposal and Concept Study Report for the competed SMEX mission, proposed ten reviews for the major milestones. Due to circumstances originating over the initial concern about the cost of the mission and spacecraft, the AIM project was required by the Independent Integrated Review Team (IIRT) to hold over fifty reviews prior to mission CDR. As shown in Figure 10, the 3 original reviews that were to encompass Systems Requirement Review (SRR), Preliminary Design Review (PDR) and the Confirmation Readiness Review activities expanded to include 29 reviews during that timeframe. [27] Although it is difficult to quantify the complete cost impact of such an increased review requirement, at minimum there was a significant disruption of project activities and progress.

\begin{tabular}{|c|c|c|c|c|}
\hline Development & Class A & Class B & Class C & Class D \\
\hline Review & $\begin{array}{l}\text { Full formal review } \\
\text { program. Either IPAO } \\
\text { external independent } \\
\text { reviews or independent } \\
\text { reviews managed at the } \\
\text { Center level with } \\
\text { Directorate participation. } \\
\text { Include formal } \\
\text { inspections of software } \\
\text { requirements, } \\
\text { design. verification } \\
\text { documents, and code. }\end{array}$ & $\begin{array}{l}\text { Full formal review } \\
\text { program Either IPAO } \\
\text { external independent } \\
\text { reviews or independent } \\
\text { reviews managed at the } \\
\text { Center level with } \\
\text { Directorate participation. } \\
\text { Include formal } \\
\text { inspections of software } \\
\text { requirements. design, } \\
\text { verification documents, } \\
\text { and peer reviews of } \\
\text { code. }\end{array}$ & $\begin{array}{l}\text { Full formal review } \\
\text { program. Independent } \\
\text { reviews managed at } \\
\text { Center level with } \\
\text { Directorate participation. } \\
\text { Include formal } \\
\text { inspections of software } \\
\text { requirements, peer } \\
\text { reviews of design and } \\
\text { code. }\end{array}$ & $\begin{array}{l}\text { Center level reviews } \\
\text { with participation of all } \\
\text { applicable Directorates } \\
\text { May be delegated to } \\
\text { Projects. Peer reviews } \\
\text { of software } \\
\text { requirements and code }\end{array}$ \\
\hline
\end{tabular}

Figure 8 - Review Requirements for Class A, B, C, D Missions as Stated in NPR 8705.4

\begin{tabular}{|l|l|c|c|c|c|c|}
\hline & \multicolumn{2}{|c|}{ Decision Authority } & \multicolumn{2}{c|}{ Technical Authority } & $\begin{array}{c}\text { Director, } \\
\text { Office of } \\
\text { Evaluation }\end{array}$ \\
\cline { 2 - 6 } & NASA AA & MDAA & NASA CE & Center Director(s) & Approve \\
\hline Programs & Approve & Approve & Approve & Approve & Approve \\
\hline Category 1 Projects & Approve & Approve & Concur & Approve & Approve ${ }^{*}$ \\
\hline Category 2 Projects & & Approve & Concur & Approve & Approve & \\
\hline Category 3 Projects & & Approve & & & \\
NASA CE = NASA Chief Engineer \\
* Only for Category 2 projects that are $\$ 250$ million or above.
\end{tabular}

Figure 9 - Convening Authorities for Standing Review Boards as Stated in NPR 7120.5E 


\begin{tabular}{|c|c|c|c|}
\hline & SRR & & 21- May \\
\hline ЩRT Plan 4/1/03 & \& CDE Cost Peer Review & WebEx & 15-Jul \\
\hline Systems Requirements Review & SOFE Cost Peer ReView - & WebEx & 16-Jul \\
\hline & 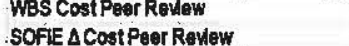 & $\begin{array}{l}\text { WebEx } \\
\text { Wubegx }\end{array}$ & 17-Jul \\
\hline MPDR / Confirmation Assessment Re & 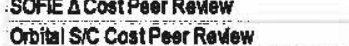 & WebEx & $\begin{array}{ll}18-\sqrt{u l} \\
21-\sqrt{u l}\end{array}$ \\
\hline Confirmation Readiness Review & $\triangle S R R$ & osc & 21 vel \\
\hline Critical Desian Review & AM Project Cost Peer Review & WebEx & 6.Aug \\
\hline Dro - & CCSRR Peer Review & Webex & 6Aug \\
\hline Pre-Environmental Review & CCSRR & LASP & 14-Aug \\
\hline Pre-Shipment Review & $\begin{array}{l}\text { SIC Peer ReViews } \\
\text { SICPDR }\end{array}$ & Oritatial & oct \\
\hline Operations Readiness Review & Soffie Peer Revewiews & soí & oet \\
\hline Mission Readiness Review & SOFE POR & SDL & $22.0 \mathrm{~d}$ \\
\hline Launch Readiness Review & CIPS \& CDE Peer Review & LASP & 23-ott \\
\hline 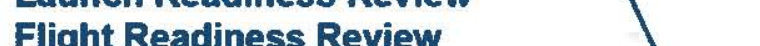 & $\begin{array}{l}\text { SOFE DPDR Action Plannling Meeting } \\
\text { CIPSICDE PDR }\end{array}$ & $\begin{array}{l}\text { SDL } \\
\text { LASP }\end{array}$ & 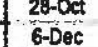 \\
\hline & Judson Detactor Fact Finding & Judson & 10-Dec \\
\hline & Bus Themal Peer Revew : & Otbital & 11-Dec \\
\hline - 3 planned reviews grew to 29 & SOFEE Peer Reviews & SOL & 13-Dec \\
\hline & $\begin{array}{l}\text { SOFE PDR } \\
\text { SIC Structure Poer Rovew }\end{array}$ & $\begin{array}{ll}\text { sol } \\
\text { Oofbital }\end{array}$ & $\begin{array}{l}\text { 13-Jan } \\
\text { 20.Jan }\end{array}$ \\
\hline & & LASP & 27 Jan \\
\hline - 50+ Reviews from 5/03 SRR to & 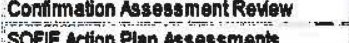 & USP & 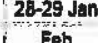 \\
\hline 11/04 MCDR Including 3 SRRs & $\begin{array}{l}\text { SOFIE Action Plan Assessments } \\
\text { Schedule Paer Reviews - Staf and IIRT }\end{array}$ & $\begin{array}{c}\text { Sol } \\
\text { Wobex } \\
\text { Wobs }\end{array}$ & 4timer \\
\hline and 2 SOFIE PDRs & Stoering Miror Peer Redew & SSG & 15-17 Mar \\
\hline & AM Confím afion Reviow & $\begin{array}{l}\text { GSFC } \\
\text { NASAHO }\end{array}$ & 19-Mar \\
\hline
\end{tabular}

Figure 10 - Expansion of Review Experienced by AIM as Presented by the AIM Principal Investigator [27]

Although the NASA ADM Project Manager also commented on the escalation of reviews that AIM experienced, he also stated the benefit of internal peer reviews in the following statements: "One type of review - peer reviews - were of significant value, particularly in the early phases of the project when technical input and critique were incorporated for a modest investment in time. Some of the most effective peer reviews were small and informal with a modest number of expert participants. On more than one occasion, however, the peer reviews were preceded by a dry run peer review to enable the design team to work issues. As these reviews become more broadly attended with increased formality, they lose the original intent. One wonders how this trend to formality might be reversed." [28]

\section{Considerations for Streamlining}

To more fully understand the possibility of streamlining reviews, the practice of two United States Air Force (USAF) organizations were investigated. Although these organizations launch a variety of different types of missions, both launch a subset of missions that are equivalent in scope to NASA Category 3 missions and, for this subset, they experience a relatively high mission success rate. It must be noted, however, that the missions developed by these organizations are primarily short term technology demonstration missions and, therefore, have different overall objectives than NASA science missions. There should be some consideration given that technology demonstrations missions may be able to allow for some liberties that a NASA Science mission may not be able to take. Given their relatively high success rate, however, an assessment of review practices of these organizations was conducted.
The Department of Defense (DOD) Space Test Program (STP) is chartered by the Office of the Secretary of Defense to serve as "...the primary provider of mission design, spacecraft acquisition, integration, launch, and on-orbit operations for DOD's most innovative space experiments, technologies and demonstrations". [29] The Space Test Program has been providing access to space for the DOD space research and development community since 1965 . The Space Test Program has a long history and welldeveloped expertise in mission design, spacecraft bus acquisition, payload integration and testing, and launch and on-orbit operations.

The Air Force Research Laboratory's Space Vehicles (AFRL/RV) Directorate leads the nation in space supremacy research and development. Their mission is to develop and transition innovative high-payoff space technologies supporting the warfighter, while leveraging commercial, civil and other government space capabilities to ensure America's advantage. [30]

Figure 11 shows the failure rate of the STP and AFRL organizations as compared to the failure rate of NASA Category 3 missions. As can be seen, the combined failure rate of STP and AFRL missions is significantly lower than for NASA missions. In the same time period from 1997 to 2011, STP and AFRL have launched fourteen missions that are relatively equivalent to NASA Category 3 missions. Of those 14 missions, only one experienced a spacecraft failure and only one experienced a launch vehicle failure for an overall failure rate of $14 \%$ as compared to the one launch vehicle and five spacecraft failures of the 23 NASA Category 3 missions launched in that same time period. Based on the relative success of their missions, Aerospace personnel supporting these organizations were asked to 
provide comments on their review process in order to identify differences STP's and AFRL's review approach relative to NASA.

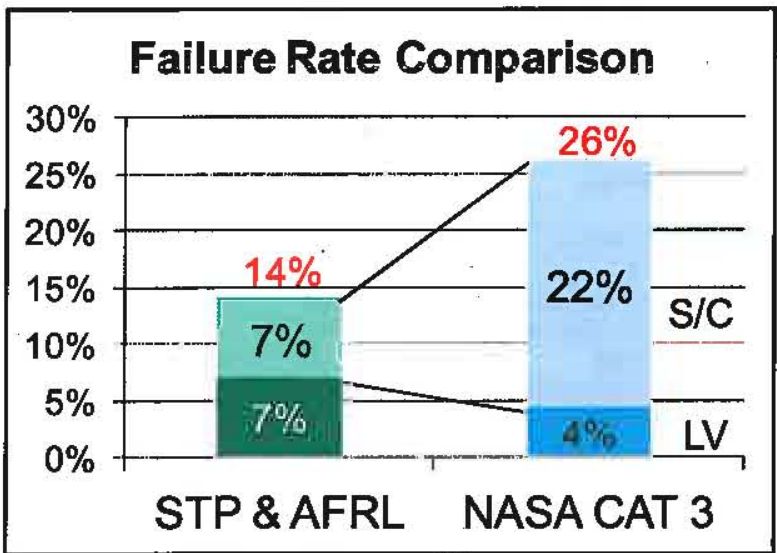

Figure 11 - Failure Rate for Equivalent Category 3 Missions

\section{STP and AFRL Experiences [31]}

When discussing the STP and AFRL review processes, it was clear that the reviews by themselves were not the primary contributing factor to the success of their missions. Both STP and AFRL have a unique, streamlined mission assurance approach that relies on identifying high risk elements early and then focusing on these risk areas with greater scrutiny while minimizing review of the lower or accepted risk items. Both STP and AFRL rely on the contractor's normal best practices while focusing on the high risk areas throughout the project while utilizing the major reviews as a discriminating gate for passage to the next phase. Although both STP and AFRL start with standard entry and exit criteria for major reviews, they streamline these criteria tailored to each mission based on the initial and continuing risk assessment. This provides for an environment where the limited review resources are focused on the items that matter the most.

STP normally conducts the following formal reviews for each spacecraft it acquires: System Requirements Review (SRR), Independent Baseline Review (IBR), Preliminary Design Review (PDR), Critical Design Review (CDR), Integration Readiness Review (IRR), Test Readiness Review (TRR), Space Flight Worthiness Certification (as part of the MRR), Pre-Ship Review (PSR), Mission Readiness Review (MRR), Flight Readiness Review (FRR), and Normal Operations Readiness Review (NORR). The PDR and CDR are usually about three days in length. Other reviews are several hours to one day in length. Reviews are normally chaired by the Program Manager, except for the MRR and NORR which is chaired by the Space Division/Space Test Program (SD/STP) Director, and the FRR which is chaired by Space and Missile Systems Center Commander (SMC/CC).

The Aerospace Corporation (Aerospace) applies a process to tailor design review criteria for STP missions. All missions,
Class A through $\mathrm{D}$, start with the design review criteria outlined in the Aerospace Space Vehicle Systems Engineering Handbook. Aerospace then sends out a tailored version of the design review criteria with the tailoring based on how much funding has invested in the project. In some cases the criteria may be significantly tailored by taking a quick look of each of the major subsystems so that there is a level of confidence that the supplying organization is following good practices After approval by STP, the list is sent to the contractor for acknowledgement that these areas will or will not be addressed in the review. At this point, the contractor is provided the opportunity to negotiate scope. Once the criteria are decided upon, Aerospace attends the review and provides verbal comments and action items. STP has approval authority at major milestones and utilizes the verbal comments and action items as input. If the project does not pass the Design Review then STP requires that either all of the liens be properly closed or requires a Delta Design Review in order to enter the next phase.

STP also relies on many information reviews conducted by the contractor as part of their normal practices. Technical Issue Reviews (TIRs) are informal. Peer Reviews of subassemblies are occasionally conducted and led by the contractors during development of the subsystems and software systems.

An Independent Readiness Review Team (IRRT), comprised of from 4-6 independent (of the program being evaluated) reviewers and Subject Matter Experts (SMEs) as needed, is organized by SMC. The IRRT is usually comprised of personnel from several organizations.

Similarly, AFRL routinely only conducts the following reviews: SRR, PDR, CDR, PSR, MRR and participates in the SMC/CC FRR. In the past few years AFRL has also begun conducting an Operations Readiness Review (ORR) before the MRR. This focuses almost exclusively on the operations strategy, on-orbit and operations risks, and operations personnel readiness. The SRR, PDR, and CDR are usually 2 days in length and are chaired by the Program Manager with AFRL/RV leadership in attendance as spectators. The MRR is specifically chaired by the AFRL/RV Director.

In addition, AFRL routinely conducts an Independent Readiness Review (IRR), comprised of 4-6 independent (of the program being evaluated) reviewers plus subject matter experts (SMEs) as needed and as directed by the AFRL/RV Director. The IRR is usually supported by The Aerospace Corporation, with SMEs usually drawn from The Aerospace Corporation's engineering group.

Peer Reviews are routinely and frequently conducted by the contractors during development of the subsystems and software systems. Occasionally Internal Design Reviews (IDRs) are conducted at the contractors; but such reviews are rare. 
AFRL minimizes their documentation and distracting reviews. The ones conducted have over the years proven their worth. They have occasionally tried to do others; but the value never justifies the expense.

In terms of documentation, AFRL again produces documentation that has been proven by AFRL to be of value. They produce SRR, PDR, CDR and PSR briefing slides but no other accompanying documentation. There is one exception: the System Requirements that are given to the contractor(s) are formally and contractually documented. There are no system specifications beyond what the contractor(s) consider in their contracts to be "normal, best practices" for them. The Government produces no such documents on AFRL projects. At the system-level, test planning is formalized but documentation is in Engineering Notebooks. Test procedures and results are published in formal, policy-compliant, short reports. A caution is that this "minimal documentation" can sometimes lead to an under-delivery of material that aids in the transitioning of technologies, which can be a hindrance to technology adoption. Design specifications (especially "as built" specs) and test reports that focus on what has been learned about the various technologies are distinctly lacking. However, it is the culture of AFRL to proceed cautiously in terms of adding tasks, reviews, or documentation without careful and controlled proof that the benefits outweigh (usually AFRL seeks "far outweighs" rather than just "outweighs") the costs (in dollars, time, and skills).

An additional, unique difference for AFRL missions includes the requirements development process. Virtually all requirements at AFRL are mutable; they are not "written in stone." The only requirements at AFRL that are typically "written in stone" are the Mission-level experiment requirements. These are the highest level of requirements and are established at the beginning of the project when it is formed. All lower-level requirements are mutable and "negotiable" throughout the programs to balance cost, schedule and technical performance. AFRL works hard to ensure that requirements do not ever force them into compromised schedules or costs.

\section{Recommendations}

Mission Assurance is a combination of many processes and factors of which the reviews and review process is a limited part. The 2010-2011 Mission Assurance Improvement Workshop (MAIW) program addressed this issue in more detail in developing the "Mission Assurance Guidelines for A-D Mission. Risk Classes" which is based on recommendations from a team comprised of government and industry team partners and interviews with different supporting organizations such as STP and AFRL. The goal of the team was to develop guidelines to define characteristic profiles for mission assurance processes for a given space vehicle risk Class (A, B, C, or D) to serve as a recommended technical baseline suitable to meet program needs based on programmatic constraints and mission needs. Appendix B2 of the MAIW document specifically relates to the review process as recommended for Class $\mathrm{C}$ and D missions - i.e., Category 3 missions - to ensure that the review process is commensurate with the level of accepted mission risk. [32] The following is a summary of the recommendations made by the MAIW relative to review requirements for Class $\mathrm{C}$ and $\mathrm{D}$ missions.

Class C Reviews Guideline-Risk-accepting Class C program reviews may not include the full suite of reviews. Early in the project-definition phase less critical reviews may be eliminated to balance cost containment against the risk of late issue identification. Planned reviews are typically documented in the project plan. Key reviews such as SRR, CDR, and MRR are generally held in compliance with contractor or industry standards. Review material generally follows standards for such reviews with some modification allowed to manage review cost. Items eliminated are perceived low risk to the project.

Class D Review Guidelines-High-risk tolerant projects typically hold only at a few key milestone reviews during their lifecycle. Key milestones include requirements definition, design determination, prefabrication, and post hardware fabrication prior to transfer to the customer. These reviews typically include a few key internal to the contractor with contractor personnel who have similar project experience. External customers may be invited but are not required to participate. Review material is less formal in content and is often less than fully compliant with industry standards for such reviews. Early planning for all projects, including Class D projects, should include a discussion regarding the reviews to be held during the project's lifecycle.

A primary lesson learned is that, prior to any review, it is beneficial for a project to perform an internal readiness review to verify that they are ready to start and complete the review at hand. In addition, prior to conducting an independent review, the development of all entrance and exit criteria for each review to determine Mission Class A-D specific entrance and exit criteria would be useful to set the expectations for that specific review. Also, required program Independent Reviews should be defined in the Project Management Plan during project kick-off. Lastly Independent Review (IR) criteria should be defined early on to understand in the context of contractor policies.

All reviews are given the following consideration:

1. Requirement:

$$
\text { - Required, Recommended, Discretionary }
$$

2. Independence:

- External, Internal, Developer

3. Completeness (see following paragraphs)

Review Completeness Guidelines for Class C MissionsOnly core mission assurance topics described in the exit criteria will be reviewed. The Independent Review Team (IRT) works with program management to determine and 
review the high and medium-high risk/mission critical areas. Interviews conducted with key players in the high and medium risk/mission critical areas. Class $\mathrm{C}$ requires the program to prove completion by review of examples, $100 \%$ physical review is not required. Tailoring of Independent Review (IR) criteria is permitted to allow review of summary analysis of evidence is acceptable through agreement between the IRT leadership and the program office. IRs are typically performed on an ad hoc basis.

Review Completeness Guidelines for Class D MissionsReviews performed only on core mission assurance required by launch safety or potentially impacting any higher-class payload (if rideshare configuration) described in the exit criteria. The IRT will work with project management to determine and review the high risk/mission critical areas. Interviews should be conducted on a subset of the key players only in the high risk/mission critical areas. Work is performed through a scaled down checklist pre-defined by agreement between the IRT leadership and the project. Class D uses word of mouth or sampling as sufficient evidence, not necessarily requiring physical review of objective evidence. Significant tailoring of IR criteria is acceptable through agreement between the IRT leadership and the project which may not include SMEs from all technical disciplines (focus is on critical requirements of the mission). The IR is mostly considered an ad hoc function.

IR requirements are stated as Required, Recommended or Discretionary. Required reviews are formally part of the project per contract requirement following an internal/external standard. Recommended reviews are highly suggested following an internal/external standard that can be tailored from the suggested levels of independence and completeness. Discretionary reviews will be at the discretion of the project, contractor and/or customer following a defined process, which, at a minimum, should include the independence and completeness levels as indicated in the matrix.

The levels of independence are stated as Externally Independent, Internally Independent and Developer Independent. Externally Independent reviewers are organizations or personnel that are technically, managerially, and financially independent of the contractor. Internally Independent reviewers are organizations or personnel that are within the contracting organization - i.e., NASA Center - that are technically, managerially, and financially independent of the project. Developer Independent reviewers are organizations or personnel that are within the contractors that are technically independent of the review subject developer team.

A summary of the recommended reviews for Class C and D missions is shown in Table 6. For Class $\mathrm{C}$ missions, CDR and MRR are the only reviews that are proposed to be required, while SRR/SDR/MDR, PDR, PSR and FRR are recommended with SIR and PER being discretionary. For Class D missions, given the high level of risk accepted and the minimal consequence for failure, no system-level reviews are proposed to be required with only CDR, PSR and MRR being recommended. All other reviews for Class $\mathrm{D}$ missions are considered discretionary. [32] Based on the MAIW recommendations presented in Table 6 , one interesting consideration is that the project's baseline confirmation review may need to be postponed until after $\mathrm{CDR}$ given that $\mathrm{CDR}$ is required for Class $\mathrm{C}$ missions, with PDR only recommended, whereas PDR is listed as fully discretionary for Class $\mathrm{D}$ missions. This would be in direct conflict with NPR 7120.5E, however, and would have to be given significant further thought.

Table 6. Recommended Review Process Streamlining for Class C and D Missions

\begin{tabular}{|l|c|c|c|c|}
\hline \multirow{2}{*}{ Review } & \multicolumn{2}{|c|}{ Class C } & Missions & \multicolumn{2}{c|}{ Class D Missions } \\
\cline { 2 - 5 } SRR/SDR/MDR & Requirement & Independence & Requirement & Independense \\
\cline { 2 - 5 } PDR & Recommended & Internally & Discretionary & Developer \\
CDR & Recommended & Developer & Discretionary & Developer \\
SIR & Required & Internally & Recommended & Internally \\
PER & Discretionary & Developer & Discretionary & Developer \\
PSR & Discretionary & Developer & Discretionary & Developer \\
MRR & Recommended & Internally & Recommended & Internally \\
FRR & Recuired & Externally & Recommended & Internally \\
\hline
\end{tabular}

\section{SUMMARY}

The study provides an assessment of NASA's Category 3 missions relative to Category 1 and 2 missions over the last 15 years. The data collected indicates that, although Category 3 missions cost less than Category 1 and 2 missions, they deliver less science and fail more often.
When looked at as a whole, however, Category 3 missions provide a cost effective acquisition approach for missions that can fit within the defined cost constraints. Simply stated, Category 3 missions are not effective for delivering much of NASA's mission portfolio but, when used in appropriate situations, they can be a very cost-effective tool 
to achieve specific science. In an effort to fully realize the benefits to a mission portfolio that includes Category 3 missions, it is recommended to leverage the review practices of the DOD STP and AFRL missions and recommendations made by the MAIW in an effort to potentially streamline the review process for Category 3 missions. As a next step, focused, streamlined mission assurance practices should also be investigated for Category 3 missions to potentially decrease the overall failure rate. This will also have a secondary, but important result of controlling cost and schedule increases. While this is a first step in the analysis, the continued success of NASA Category 3 missions is important for NASA's cost-constrained future.

\section{ACKNOWLEDGEMENTS}

The authors would also like to acknowledge all of the individuals from The Aerospace Corporation who have contributed to the data collection efforts and the discussion of the results. These include: A. Clark Keith, David D Voelkel, Sam Myers Sims, Lisa Berenberg and Gary Hendel. The authors would also like to acknowledge Gail Johnson-Roth, Edward Hume. Jean-Claude Inauen, Mark Oja, and Brian Shaw for their contributions to Appendix B2 of the MAIW document which was very heavily utilized in this paper. Additionally, Debra Emmons, and Francesco Bordi also provided feedback and discussion that was invaluable in guiding the final study results. A special thanks to Param Nair, NASA Goddard Space Flight Center for some specific, excellent feedback on the draft version of the paper.

\section{REFERENCES}

[1] Committee on Earth Studies, Space Studies Board, National Research Council, The Role of Small Satellites in NASA and NOAA Earth Observation Programs", Committee on Earth Studies, Space Studies Board, National Research Council, 2000.

[2] Committee on Principal-Investigator-Led Missions in the Space Sciences, National Research Council, "PrincipalInvestigator-Led Missions in the Space Sciences" Committee on Principal-Investigator-Led Missions in the Space Sciences, National Research Council, 2006, http://www.nap.edu/catalog/11530.html.

[3] Committee on Heliophysics Performance Assessment; National Research Council, "A Performance Assessment of NASA's Heliophysics Program", Committee on Heliophysics Performance Assessment; National Research Council, 2009, http://www.nap.edu/catalog/12608.html.

[4] "NASA Space Flight Program and Project Management Requirements", NASA Procedural Requirements (NPR7120.5E), 14 August 2012.

[5] "Risk Classification for NASA Payloads", NASA
Procedural Requirements NPR-8705.4, 14 June 2004.

[6] "Evaluating Small Satellites: Is The Risk Worth It?", SSC99-IIA-1, 1999 Small Satellite Conference, 23 August 1999, Todd Mosher, Robert Bitten, Norm Lao, Eric Mahr, Riaz Musani.

[7] NASA Release: 98-109. "Lewis Spacecraft Failure Board Report Released", Florida Today, June 23, 1998.

[8] Lewis Spacecraft Mission Failure Investigation Board Report. February 12, 1998.

[9] NASA News Release. "Investigation: Design errors caused WIRE failure." June 25, 1999.

[10] Boston University Press Release. "Student-Built TERRRIERS Satellite Experiencing Difficulties." May 19, 1999.

[11] Mars Climate Orbiter- Phase I Report November 1999, Mishap Investigation Board.

[12] Report on Loss of the Mars Polar Lander and Deep Space 2 Missions - March 22, 2000, JPL Special Review Board.

[13] CONTOUR Mishap Investigation Board Report, NASA, May 1, 2003.

[14] J. Ray, "NASA Cancels Clark Satellite Mission." Florida Today, February 25, 1998.

[15] FY2000 NASA Budget Estimates, pg. SI-33, NASA Major Development Programs.

[16] FY2001 NASA Budget Estimates, pg. SAT-1-64, Space Science Budget Details.

[17] FY2003 Office of Earth Science Budget Estimates Summary, pg. SAT 3-85.

[18] FY2002 Office of Space Science Budget Estimates Summary, pg. SAT 1-30.

[19] FY2003 Office of Space Science Budget Estimates Summary, pg. SAT 1-42.

[20] "NASA cancels space science mission", Space Today, 4 June 2003, http://www.spacetoday.net/Summary/1695.

[21] Jane's Space Systems and Industry, 5 April 2006, http://articles.janes.com/articles/Janes-Space-Systems-andIndustry/CATSAT-STEDI-series-United-States.html.

[22] FY2008 NASA Budget Estimates, pgs. SMD-239, 240.

[23] NASA Press Release, Sept. 21, 2001, http://www.nasa.gov/newsinfo/quiktoms.html. 
[24] OCO Mishap Investigation Results, NASA, July 16, 2009.

[25] Space News, http://www.spacenews.com/launch/110421-orbital-launchfailure-review-nears-conclusion.html, 21 April 2011.

[26] "Criteria for Flight Project Critical Milestone Reviews", GSFC-STD-1001, Baseline Release 19 February 2005.

[27] J.M. Russell III, "AIM Mission Responses to the Unexpected", presented at The PI-Team Masters Forum, April 28, 2010.

[28] M.T. McGrath, "The Aeronomy of Ice in the Mesosphere Mission", presented at the IEEE Aerospace Conference, March 2009.

[29] Space Test Program Fact Sheet, 25 January 2007, http://www.kirtland.af.mil/library/factsheets/factsheet.asp?i $\mathrm{d}=6878$.

[30] Air Force Research Laboratory Fact Sheet, 3 Nov 2011, http://www.kirtland.af.mil/library/factsheets/factsheet.asp?i $\mathrm{d}=7873$.

[31] Internal Aerospace Corp. Memo, A Clark Keith, and David D Voelkel, 8 October 2012.

[32] "Mission Assurance Guidelines for A-D Mission Risk Classes", Gail Johnson-Roth, 3 June 2011, Aerospace Report TOR-2011(8591)-21.

\section{BIOGRAPHY}

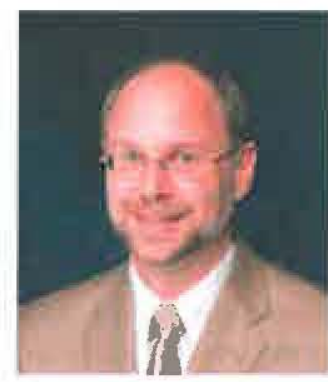

Robert Bitten is a Principal Engineer at The Aerospace Corporation and has conducted independent cost estimates for NASA proposal evaluations and independent assessments for $a$ variety of different NASA missions and organizations. He is a winner of the President's Award, The Aerospace Corporation's highest honor, for his effort in assessing the cost effectiveness of different alternatives in the in the Hubble Space Telescope Remote Servicing Module Analysis of Alternatives. He also won the 2007 NASA Cost Estimating Support Contractor of the Year Award that is awarded to recognize an individual who has provided "outstanding contractor support to the NASA cost estimating community and significantly contributed to the field of cost estimating."

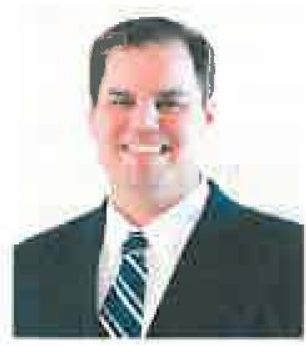

Steve Shinn is Deputy Director for Planning \& Business Management for the Flight Projects Directorate at NASA Goddard Space Flight Center. In this role, he is responsible for the strategic initiatives and organizational processes that must be planned and deployed throughout the Directorate in order to successfully support its mission in matters relating to business, resource management, performance management, organizational staffing, workforce development, human resources processes, core capabilities, and physical assets. Prior to NASA GSFC, he was Supervisor of Operations Management at The Johns Hopkins University Applied Physics Laboratory Space Department where he managed a group of more than 40 engineering and business professionals and was responsible for business operations supporting the Civilian Space and National Security Space Business Areas. He has held various management positions in pricing, cost management, estimating, operations management, software management, EVMS, and scheduling. He is also an instructor in Project Planning and Control at The Johns Hopkins University Whiting School of Engineering.

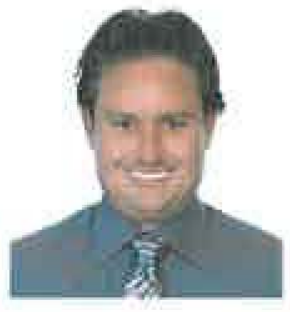

Eric Mahr is a Senior Engineering Specialist in the Space Architecture Department at The Aerospace Corporation. His expertise is in spacecraft and architecture development. He has worked on a number of architecture and mission developments, studies and evaluations for NASA, the Air Force, and commercial organizations. He has a B.S. in Aerospace Engineering from the University of Arizona and a M.S. in Aerospace Engineering from the University of Colorado. 


\section{APPENDIX A}

\section{LIST OF NASA MISSIONS INCLUDED IN STUDY}

\begin{tabular}{|c|c|c|c|}
\hline NASA Mission: & Fught & Earesur & Elass: \\
\hline Lewis (1) & Aug-97 & CAT 3 & $\mathbf{C}$ \\
\hline Explorer ACE (3) & Aug-97 & CAT 2 & C \\
\hline Cassini Orbiter (1) & Oct-97 & CAT 1 & A \\
\hline Discovery Lunar Prospector (1) & Jan-98 & CAT 3 & $\mathbf{C}$ \\
\hline Explorer UNEX SNOE (1) & Feb-98 & CAT 3 & D \\
\hline Explorer SMEX TRACE (3) & Apr-98 & CAT 3 & $C$ \\
\hline NMP Deep Space (DS1) & Oct-98 & CAT 3 & C \\
\hline Explorer SMEX SWAS (4) & Dec-98 & CAT 3 & C \\
\hline Mars Clımate Orbiter (1) & Dec-98 & CAT 3 & $\mathrm{C}$ \\
\hline Mars Polar Lander (1) & Jan-99 & CAT 2 & C \\
\hline DIscovery STARDUST (1) & Feb-99 & CAT 2 & B \\
\hline Explorer SMEX WIRE (5) & Mar-99 & CAT 3 & C \\
\hline Landsat 1 (7) & Apr-99 & CAT 1 & B \\
\hline Explorer UNEX TERRIERS (2) & May-99 & CAT 3 & D \\
\hline EOS QuikSCAT (1) & Jun-99 & CAT 3 & $\mathrm{C}$ \\
\hline Explorer MIDEX FUSE (0) & Jun-99 & CAT 2 & C \\
\hline Chandra (1) & Jul-99 & CAT 1 & A \\
\hline EOS Terra (1) & Dec-99 & CAT 1 & B \\
\hline EOS ACRIMSAT (1) & Dec-99 & CAT 3 & C \\
\hline Explorer MIDEX IMAGE (1) & Mar-00 & CAT 2 & C \\
\hline Explorer HETE (2) & Oct- -00 & CAT 3 & C \\
\hline NMP EO (1) & Nov-00 & CAT 2 & C \\
\hline 2001 Mars Odyssey (2) & Apr-01 & CAT 2 & B \\
\hline Explorer MIDEX MAP (2) & Jun-01 & CAT 2 & C \\
\hline Discovery Genesis (1) & Aug-01 & САT 2 & B \\
\hline QuikTOMS (1) & Sep-01 & CAT 3 & C \\
\hline STPP TIMED (1) & Dec-01 & CAT 2 & C \\
\hline Explorer SMEX HESSI (6) & Feb-02 & CAT 3 & C \\
\hline ESSP GRACE (1\&:2) & Mar-02 & CAT 3 & C \\
\hline EOS Aqua (1) & May-02 & CAT 1 & B \\
\hline Discovery CONTOUR (1) & Jul-02 & CAT 3 & B \\
\hline EOS ICESat (1) & Jan-03 & CAT 2 & B \\
\hline Explorer UNEX CHIPSAT (3) & Jan-03 & CAT 3 & D \\
\hline EOS SORCE (1) & Jan-03 & CAT 3 & $\mathrm{C}$ \\
\hline Explorer SMEX GALEX (7) & Apr-03 & CAT 3 & C \\
\hline 2003 Mars Explor Rov (MER-A) & Jun-03 & CAT 1 & B \\
\hline Origins SIRTF (1) & Aug-03 & CAT 1 & B \\
\hline $\mathrm{GP}-\mathrm{B}(1)$ & Apr-04 & CAT 1 & B \\
\hline EOS Aura (1) & Jul-04 & CAT 1 & B \\
\hline Discovery MESSENGER (1) & Aug-04 & CAT 2 & B \\
\hline Explorer MIDEX SWIFT (3) & Nov-04 & CAT 2 & C \\
\hline Discovery Deep Impact (1) & Jan-05 & CAT 2 & B \\
\hline 2005 Mars Rec Orb (1) & Aug-05 & CAT 1 & B \\
\hline New Frontiers New Horizons (1) & Jan-06 & CAT 1 & B \\
\hline ESSP CLOUDSAT (1) & Apr-06 & CAT 2 & $\mathrm{C}$ \\
\hline STPP STEREO (A\&B) & Oct-06 & CAT 2 & C \\
\hline Explorer MIDEX THEMIS (1-5) & Feb-07 & CAT 3 & C \\
\hline Explorer SMEX AIM (8) & Apr-07 & CAT 3 & C \\
\hline Phoenix (1) & Aug-07 & CAT 2 & B \\
\hline Discovery DAWN (1) & Sep-07 & CAT 2 & B \\
\hline
\end{tabular}

\begin{tabular}{|c|c|c|c|}
\hline MASA MVESTLMS: & Eundy & CEk+BMIN & Jass \\
\hline GLAST (1) & Jun-0̈8 & CAT 2 & B \\
\hline Explorer SMEX IBEX (10) & Oct-08 & CAT 3 & C \\
\hline ESSP OCO (1) & Feb-09 & CAT 2 & C \\
\hline Discovery Kepler (1) & Mar-09 & САT 2 & B \\
\hline LCROSS (1) & Jun-09 & CAT 3 & D \\
\hline Lunar Recon Orbiter (1) & Jun-09 & CAT2 & C \\
\hline Explorer MIDEX WISE (5) & Dec-09 & CAT 2 & $\mathrm{C}$ \\
\hline LWS SDO (1) & Feb-10 & CAT 1 & B \\
\hline EOS Glory (1) & Mar-11 & САT 2 & C \\
\hline GRAIL & Sep-11 & САТ 2 & B \\
\hline JUNO & Aug-11 & CAT 1 & B \\
\hline MSL & Nov-11 & CAT 1 & B \\
\hline GLAST (1) & Jun- 08 & САT 2 & B \\
\hline Explorer SMEX IBEX (10) & Oct-08 & CAT3 & C \\
\hline ESSP OCO (1) & Feb-09 & CAT 2 & $\mathrm{C}$ \\
\hline Discovery Kepler (1) & Mar-09 & CAT 2 & B \\
\hline LCROSS (1) & Jun-09 & CAT 3 & D \\
\hline Lunar Recon Orbiter (1) & Jun-09 & CAT 2 & $\mathrm{C}$ \\
\hline Explorer MIDEX WISE (5) & Dec-09 & САТ 2 & C \\
\hline LWS SDO (1) & Feb-10 & CAT 2 & B \\
\hline EOS Glory (1) & Mar-11 & CAT 2 & C \\
\hline GRAIL & Aug-11 & CAT 2 & B \\
\hline JUNO & Aug-11 & CAT 1 & B \\
\hline MSL & Nov-11 & CAT 1 & B \\
\hline
\end{tabular}

APPENDIX B

\section{LIST OF DOD MISSIONS INCLUDED IN STUDY}

\begin{tabular}{|l|r|}
\hline STP Missions & launch \\
\hline STEP M4 & 1997 \\
\hline TSX-5 & 2000 \\
\hline PICOSat & 2001 \\
\hline Coriolis & 2003 \\
\hline STPSat-1 & 2007 \\
\hline CNOFS & 2008 \\
\hline STPSat-2 & 2010 \\
\hline
\end{tabular}

\begin{tabular}{|l|r|}
\hline AFRL Missions & Launch \\
\hline MightySat-1 & 1999 \\
\hline Warfighter & 2001 \\
\hline MightySat-2 & 2002 \\
\hline XSS-10 & 2003 \\
\hline XSS-11 & 2005 \\
\hline TacSat-2 & 2007 \\
\hline TacSat-3 & 2009 \\
\hline
\end{tabular}




\section{APPENDIX C}

\section{FAILURe RATe CASE StUdies}

The following includes a short summary of failed and cancelled/non-confirmed missions that is largely taken from [6] but has been updated with additional cases. The original references are included for the reader to do further research into the causes.

\section{Case Studies for Spacecraft Failures}

Lewis was launched on August 23, 1997 and re-entered the atmosphere on September 28 due to catastrophic failure. The goal of Lewis, to demonstrate advanced science instruments and new technologies for measuring changes in topography, was never reached as the spacecraft entered a flat spin in orbit resulting in loss of power to the solar arrays and eventual battery power discharge. Ground controllers lost contact on August 26. The attitude control system design had been adapted from a system used on the Total Ozone Mapping Spectrometer/Earth Probe (TOMS/EP) spacecraft. Investigation into the attitude control system found that insufficient analysis had been done to adapt this design to a different spacecraft spin-axis orientation. [7][8] Lack of knowledge about the behavior of the spacecraft in orbit resulted in rotational perturbations, which eventually led to an uncontrolled spin. Lewis was a Category 3 mission.

WIRE, the Wide-Field Infrared Explorer, launched on March 4, 1999 suffered catastrophic failure due to design error and analysis. Known characteristics about a component in the instrument electronic box were not considered in depth, leading to an electrical power surge reaching the explosive devices at startup. [9] The activation of the pyrotechnics resulted in premature ejection of the telescope's cover resulting in exposure of the frozen hydrogen (used as a coolant) and the telescopes infrared detectors to the sun. As the telescope heated, the hydrogen converted to gas and expelled entirely within a period of 48 hours from launch. Without the necessary cooling, the scientific mission was considered a loss. WIRE was a Category 3 mission.

TERRIERS, the Tomographic Experiment using Radiative Recombinative Ionospheric EUV and Radio Sources satellite, built under the NASA Student Explorer Demonstration Initiative (STEDI), launched on May 18, 1999. An orientation problem with the spacecraft to allow the solar arrays full exposure to the Sun resulted in battery discharge. [10] The orientation error could be attributed to possible errors in the attitude control system software. TERRIERS was a Category 3 mission.

MCO, the Mars Climate Orbiter built by Lockheed Martin under the JPL Mars Surveyor '98 contract, was designed to serve as a communication relay for Mars Polar Lander and monitor weather around Mars. Launched on December 11, 1998, MCO was lost during the Mars Orbit Insertion phase.
The root cause of failure of the MCO mission was determined to be due to the use of English units rather than metric units in a ground software file. [11] The software file was used for trajectory modeling. This software design error coupled with over compensation of angular momentum desaturation during mission operations resulted in MCO's altitude at Mars insertion being $170 \mathrm{~km}$ lower than planned. [11] The investigation board concluded that either MCO was destroyed upon entering Mars' atmosphere or re-entered space leaving Mars' atmosphere. Software design error and lack of appropriate levels of program management / system engineering processes were also cited as contributing causes to the MCO failure. MCO's cost constraints and place it within the Category 3 mission classification.

MPL, the Mars Polar Lander, designed to study volatiles and climate history, was also built by Lockheed Martin under the JPL Mars Surveyor '98 contract. MPL was launched on January 3,1999, and the anticipated communication between MPL and Earth during touchdown never occurred. This loss of communication initiated a recovery effort to try and communicate with MPL, with no success. Due to the lack of flight data available, the most probable cause as to the loss of MPL was determined to be due to the an early shutdown of MPL's descent engines during the touchdown phase. [12] The possible destruction of MPL was traced back to a software design error in the landing sensors. Lean program management and lack of key system engineering processes, in addition to a severely cost-constrained program exacerbated the situation leading up to the loss of MPL. MPL's cost, which due to lander development was higher than for MCO, would have placed the mission in Category 2.

CONTOUR, the Comet Nucleus Tour, launched on July 3 , 2002 with the intended purpose of visiting multiple comets to perform a variety of investigations on the comet material. The spacecraft remained in Earth orbit until August 15, 2002, when a solid rocket motor was fired to place the spacecraft on the trajectory to the first target. Communications were not possible during the firing and the operations team expected to regain contact with the spacecraft approximately 45 minutes after firing. Contact was never made. After numerous contact attempts with no success, the mission was declared lost on December 20, 2002. While a number of reasons for the loss of the mission could not be completely ruled out, the probable cause was found to be overheating of the spacecraft from the solid rocket motor exhaust plume. The solid rocket motor was embedded within the structure of the spacecraft, as opposed to the typical external mounting, and the effect of the heating on the whole of the spacecraft from the exhaust plume was not correctly analyzed. It is possible that the heating led to catastrophic degradation of the structural integrity of the spacecraft. [13] The low cost implementation of CONTOUR designates the mission as Category 3. 


\section{Case Studies for Cancelled Missions}

The Clark spacecraft never made it to launch due to cancellation of the program in February 1998. The primary goal of Clark was to produce black and white stereo images with resolution up to $3 \mathrm{~m}$. Primary reasons for cancellation of the program can be attributed to a combination of concerns about cost overruns, payload health, and an uncertain launch schedule. Projection of cost to complete the mission showed a cost overrun of 15 percent. NASA had spent $\$ 55$ million for the budgeted $\$ 49$ million mission. [14] The instrument to be used on Clark had been sitting on the ground longer than had been expected, leading to reservations about its operation and health while in orbit. Lastly, scheduling conflicts with the Lockheed Martin Athena program resulted in further postponement of the mission. Many of the concerns leading to the cancellation of the Clark mission related to top-level requirements for the mission. Clark would have been a Category 3 mission.

ST-4, the Champollion/Comet Lander Space Technology-4 mission, was selected as a New Millennium Program (NMP) mission in 1998. ST-4 was planned to travel to, land on, and study comet 46P/Tempel 1 and (potentially) return a sample to Earth. [15] The mission was not approved to proceed to development in 1999 due to budgetary constraints caused by overruns in other mission. [16] ST-4, due to its expense, would have been a Category 2 mission.

VCL, the Vegetation Canopy Lidar (VCL) mission was selected in March 1997 as one of the first two Earth System Science Pathfinder (ESSP) missions. VCL was designed to utilize a multi laser Light Detection and Ranging (LIDAR) instrument to globally map the three- dimensional structure of the Earth's vegetation. Due to significant developmental difficulties with the instrument leading to projected cost growth, NASA decided to discontinue funding VCL in FY2001. [17] VCL would have been a Category 3 mission.

IMEX, the Inner Magnetosphere Explorer (IMEX) mission was selected in September 1998 as one of the first set of UNEX missions. IMEX was to have studied the response of Earth's Van Allen radiation belts to variations in the solar wind. In September 1998 IMEX entered Phase A, which was continued throughout FY99 due to launch vehicle unavailability. IMEX was to have entered Phase B in FY 2001 but, in January 2001, the project was not confirmed for preliminary design due to cost growth. [18] IMEX would have been a Category 3 mission.

FAME, the Full Sky Astrometric Mapping Explorer mission, was selected as a Medium Explorer (MIDEX) mission in October 1999. FAME was planned as an astrometric satellite designed to determine, with unprecedented accuracy, the positions, distances, and motions of 40 million stars within our galactic neighborhood. FAME was designed to measure stellar positions to less than 50 microarcseconds. The mission was not approved to proceed to development in early FY 2002, due to unacceptable cost growth, primarily in the instrument, as identified at the Confirmation Review. [19] FAME, being a MIDEX mission, would have been a Category 2 mission.

SPIDR, the Spectroscopy and Photometry of the IGMs Diffuse Radiation (SPIDR) mission was a SMEX mission selected in July 2002. SPIDR was designed to map concentrations of extremely hot gas located in filaments between galaxies; astronomers hypothesize that this gas may constitute some of the dark matter thought to exist in the universe. NASA cancelled the mission after determining that the mission's main instrument would not be as sensitive as advertised. [20] SPIDR would have been a Category 3 mission.

CATSAT, the Co-operative Astrophysics and Technology Satellite, was selected under the STEDI program to investigate the distance and polarization of gamma bursts. The University of New Hampshire satellite was scheduled to launch in 2002 but the project was cancelled due to cost escalation. [21] CATSAT would have been a Category 3 mission.

SIM, the Space Interferometry Mission, was a mission initially recommended by the 1991 Astronomy and Astrophysics Survey Committee as the Astrometric Interferometry Mission. SIM was to implement breakthroughs in precision control technology to measure the positions and distances of stars with unprecedented precision which could determine which small, rocky planets are in the habitable zones. Although development started in October 1998, there were substantial technology development hurdles to overcome. Although SIM met the majority of ground technology demonstrations, the mission was not approved to proceed to implementation in 2008 due to budgetary constraints with the Astrophysics budget allocation. [22] SIM, due to its expense and importance, would have been a Category 1 mission.

\section{Case Studies for Launch Vehicle Failures}

QuikTOMS, the Quick Total Ozone Mapping Spectrometer, launched on September 21, 2001 on a Taurus was to monitor ozone in the Earth's atmosphere. The launch vehicle briefly went out of control soon after the second stage ignited. Though the launch vehicle got back on course and deployed the spacecraft, the loss of the momentum during the boost phase meant that the spacecraft did not reach the correct altitude and velocity to stay in Earth orbit. The spacecraft fell back to Earth over the Indian Ocean. The loss of control was traced to the thrust vector control actuator on the second stage which did not move as designed when the stage ignited. [23] QuikTOMS was a Category 3 mission.

OCO, the Orbiting Carbon Observatory, launched on February 24, 2009 on a Taurus XI launch vehicle to make measurements to increase the understanding of carbon dioxide concentrations in the Earth's atmosphere. The mission was lost when the launch vehicle payload fairing 
failed to separate. The spacecraft separated from the third stage, but was still contained with the payload fairing. Due to the excess mass of the payload fairing, the spacecraft did not attain the correct altitude or velocity to remain in Earth orbit and fell back to Earth over the Pacific Ocean. The failure of the payload fairing to separate was traced to the design/operation of the separation system, though a specific failure mechanism could not be identified. [24] OCO was a Category 3 mission.

Glory launched on March 4, 2011 on a Taurus XL launch vehicle to study the role of aerosols on with respect to the Earth's climate. The mission was lost when the launch vehicle payload fairing failed to separate. The spacecraft, contained within the payload fairing, never made it to orbit and splashed down in the Pacific Ocean. [25] Glory was a Category 2 mission. 